\title{
The Challenge of Malignancies in HIV-1, Beyond Immune Activation and Back to Decreased Immune Surveillance
}

\section{Daniel O Griffin*}

Department of Biochemistry and Molecular Biophysics, Columbia University Medical Center, New York, USA

\begin{abstract}
The HIV-1 epidemic continues around the world, but also in the United States where we continue to see approximately 50,000 new diagnosis of HIV-1 infection each year. It is estimated that there are currently more than 1.2 million individuals in the United States living with HIV-1 infection, with 12.8\% unaware of their infection. Effective therapy for HIV-1 is allowing infected individuals to have greater life expectancies. We now have an older aging population infected with HIV-1, reaching ages where diseases such as malignancy are increased in incidence. Even compared to age matched peers there is clearly an excess of malignancies affecting the HIV-1-infected population. Malignancies are now the most common cause of death for patients in the United States living with HIV-1 infection. B-cell malignancies are the most common malignancy accounting for death in HIV-1 infected patients in the United States. It is not clear that all that we have come to understand regarding B-cell lymphomas applies to the lymphomas developing in the HIV-1-infected population. It is particularly important to understand the factors leading to and molecular disturbances involved in these lymphomas developing in the HIV-1-infected population as they appear to be increasing in frequency and characterized by aggressive courses with short median survival times. Although much attention has focused on the chronic immune activation hypothesis of cancer in HIV-1 infection, this article explores the possible contribution of decreased immune surveillance and exposure to highly active antiretroviral medications to the development of B-cell lymphomas in HIV-1-infected patients.
\end{abstract}

Keywords: HIV-1; Malignancy; Lymphoma; B-cell: Integrase inhibitor; Protease inhibitor; Non-nucleoside reverse transcriptase inhibitor; HIV-1; HIV

\section{Background}

The HIV-1 epidemic continues with 50,000 new diagnosis of HIV1 infection each year in the United States and is now characterized by an aging population of 1.2 million individuals in the United States living with HIV-1 infection who are reaching ages where diseases such as malignancy are increased in incidence [1-6]. Malignancies, particularly B-cell malignancies, are now the most common cause of death for patients in the United States living with HIV-1 infection, and is a significant factor driving 30 day hospital readmissions [7-10]. Currently available highly active antiretroviral therapy (HAART) is able to suppress viral replication, maintain CD4 T-cell levels and increase life expectancy in the majority of HIV-1-infected individuals [11,12]. $\mathrm{B}$ cell lymphoma is the most common of the malignancies responsible for cancer mortality in the current HAART treated HIV-1-infected population [8,13-15]. Although the genetic basis of and heterogeneity of B-cell lymphomas has been extensively studied in the HIV-negative population, much remains to be learned about the genetics of and causes underlying B-cell lymphomas in HIV-1-infected individuals [16]. In addition to a possible oncogenic milieu developing as a consequence of the chronic immune activation present in many HIV-1-infected individuals it is possible that loss of immune surveillance or exposure to certain medications contained in modern HAART regimens is driving the process $[17,18]$.

\section{Malignancies in HIV-1-uninfected patients}

Six hallmarks of cancer have been suggested as essential for tumor development and dissemination [19,20] Along with the six suggested pillars of oncogenesis: sustained proliferative signaling, evasion of growth suppressors, resistance to cell death, induction of angiogenesis, acquisition of replicative immortality, and activation of invasion and metastasis, immune evasion may be a critical seventh pillar of oncogenesis [19-21] (Figure 1). In many ways the first six hallmarks of cancer seem very tumor-centric. From the perspective of the host these are import checkpoints that must be maintained for homeostasis. The host's immune system, however, is the last defense of the host against a cell that has lost the critical controls and transformed into a malignancy [21]. Advances, in the understanding of malignancies, have led to an expanded model with 10 hallmarks of cancer that now includes immune evasion [20].

Advances in the understanding of cancer biology have established the importance of immune evasion as a critical feature of malignancies $[19,20]$. Immune evasion is critical for the success of all malignancies, including B-cell lymphomas, in HIV-1-uninfected individuals [21]. Without immune evasion malignant cells could be recognized by innate and adaptive cells of the immune system and cleared or destroyed [22]. There is thus a tremendous selective pressure for successful immune evasion placed on evolving tumor cells that is evident in both the genome and in the tumor exome of tumors developing in immunocompetent hosts [23].

\section{Immune evasion by malignancies in HIV-1-infected Patients, the seventh pillar of oncogenesis}

Unfortunately early in HIV-1 infection there is a significant and

*Corresponding author: Daniel O Griffin, Department of Biochemistry and Molecular Biophysics, Columbia University Medical Center, HHSC 1310c, 701 West 168th Street, New York, NY 10032, USA, Tel: 212-305-3794; Fax: 212-3055106; E-mail: dg2810@columbia.edu

Received November 30, 2015; Accepted January 08, 2016; Published January 15,2016

Citation: Griffin DO (2016) The Challenge of Malignancies in HIV-1, Beyond Immune Activation and Back to Decreased Immune Surveillance. J AIDS Clin Res 7: 535. doi:10.4172/2155-6113.1000535

Copyright: ( 2016 Griffin DO. This is an open-access article distributed under the terms of the Creative Commons Attribution License, which permits unrestricted use, distribution, and reproduction in any medium, provided the original author and source are credited. 


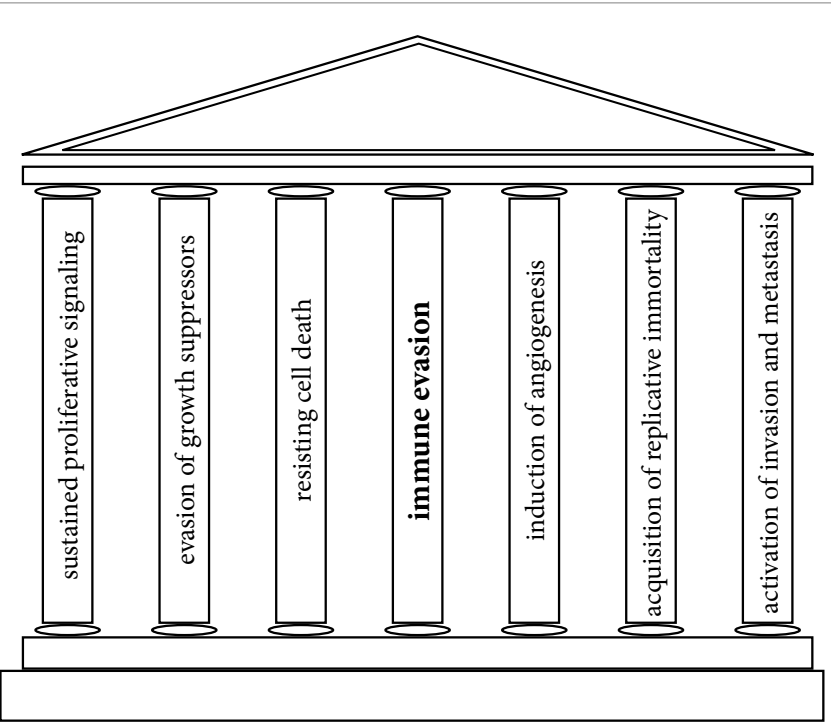

Figure 1: The seven pillars of oncogenesis. The current six pillars of oncogenesis sustained proliferative signaling, evasion of growth suppressors, resisting cell death, induction of angiogenesis, acquisition of replicative immortality, and activation of invasion and metastasis with the addition of the critical seventh pillar of immune evasion.

permanent loss of mucosal immune cells and permanent damage to the immune system despite rebound of CD4 counts from initial nadirs [24]. It is thus not surprising that, in addition to malignancies driven by oncogenic pathogens, there is an increased incidence of many malignancies not associated with oncogenic pathogens in HIV-1infected patients [6]. The barrier posed by the immune system in HIV1-uninfected individuals may be less formidable in the HIV-1-infected population.

Immune evasion may be accomplished through avoidance of detection by the immune system, neutralization of immune cells, induction of tolerance, and/or modulation of the immune response. In many malignancies immune evasion involves reduction in the surface expression of antigen presentation molecules [25]. Avoidance of detection by the immune system can be accomplished by a decrease in the antigen presentation by MHC class I antigens. This may be due to defects in the machinery that processes aberrant proteins and delivers MHC class I molecules with contained peptides to the cell surface $[26,27]$. An interesting aspect of B cell malignancies is that the malignant cells also serve as professional antigen presenting cells with inherent expression of MHC class-II molecules [28]. Decreased class II expression is associated with decreased immune response and poor prognosis [29]. Diffuse large B-cell lymphoma (DLBCL) is also often characterized by down regulation of MHC-II molecules, HLA-DR and HLA-DR, which correlates with T cell infiltration and is likely a strategy for immune evasion [30].

Malignancies may avoid destruction by the immune system by neutralizing host immune cells. B-cell lymphomas have been demonstrated to express elevated levels of Fas ligand (FasL) which can trigger Fas mediated apoptosis of cytotoxic T lymphocytes [31]. Malignancies may also accomplish neutralization of host immune cells through expression of programmed death ligand 1 (PDL1) or programmed death ligand 2 (PDL2), which reduces the proliferation of host T-cells [32].

Certain malignancies, such as B-cell lymphomas, both Hodgkins and non-Hodgkins lymphomas, also appear to have the ability to influence the surrounding tumor microenvironment and induce proliferation of tumor tolerant $\mathrm{CD}^{+} \mathrm{CD}^{+} 5^{+} \mathrm{FoxP}^{+}$T-regulatory cells [33-35]. Although it remains unclear how these T-regulatory cells are induced, they do appear to secrete IL-10 and interfere with the generation of anti-tumor Th17 cells. Myeloid derived regulatory cell induction appears to be another critical component of the B-cell lymphoma immune evasion strategy and is associated with poor overall survival [36-38]. Not only are malignant cells modulating immune cells toward an immune-tolerant phenotype but they are also attracting immunosuppressive cells to the tumor microenvironment with chemoattractant chemokines such as CCL2/MCP-1, CXCL12, CCL22, CCL5, CCL17/TARC, and fractalkine/CX3CL1 [21].

Malignancies also have the ability to express immune modulatory molecules and produce a number of secreted or soluble factors that facilitate their immune evasion. Through malignant cell expression of Galectib-1, T cells are polarized toward a Th2 phenotype and $\mathrm{T}$ cell proliferation and IFN- $\gamma$ production is decreased [39]. Prostaglandin E2 can also be produced by certain lymphomas and can influence both CD4+ T-cells and B-cells [40,41]. Malignancies are able to evade natural killer (NK) cells by the secretion of the decoy protein NKG2D which prevents activation through a surface NK cell receptor $[42,43]$. Serum sIL-2Ra may be produced and interfere with IL-2 mediated T cell activation in certain contexts $[44,45]$.

In addition to the induction of suppressive cells that secrete immunosuppressive cytokines, certain malignancies are often capable of secreting immunosuppressive cytokines such as IL-10 and IL-6 themselves [46]. Some B-cell lymphomas may in fact be developing from subsets of B cells that spontaneously or with appropriate stimulation secrete IL-10 [47,48]. Analysis of B-cell chronic lymphocytic leukemia cells has demonstrated similarities to IL-10 producing regulatory B10 cells and innate IL-10 spontaneously secreting B cells [49-53]. TGF- $\beta$ is another immunomodulatory molecule produced in both membranes bound and secreted forms by malignancies as part of their immune evasion [54].

With the importance of evading a competent immune system demonstrated and our understanding of how this is achieved, the appearance of an increased incidence not associated uncontrolled activity of an oncogenic virus starts to seem more expected than surprising. Currently, however, little is known about whether the decreased immune pressure present in an HIV-1-infected patient is evident in the degree and type of immune evasive changes. Further investigation is needed in order to discover how much decreased immunity contributes to the excess frequency of cancers and cancer related mortality.

\section{The impact of antiretroviral therapy on immune surveillance and malignancy development}

In addition to the fact that lower levels of $\mathrm{CD} 4^{+} \mathrm{T}$ cells and decreased competence of the immune system change the environment in which tumors develop, there are also a number of chronic medications that most HIV-1-infected individuals are currently taking. There are now a large number of antiretroviral medications in use for the treatment of HIV-1 infection [55]. Although these medications are available and effective, a growing percent of $\mathrm{HIV}$-1-infected individuals are dying due to malignancies despite suppressed HIV-1 viral load and preservation of CD4 count and percentage $[8,9,15]$. It is usual and necessary for a number of these medications to be used together in a 'cocktail' to successfully treat HIV-1 infection [55]. 
The classes of medications used to treat HIV-1 infection are; nucleoside/nucleotide reverse transcription inhibitors (NRTIs), non-nucleoside reverse transcription inhibitors (NNRTIs), protease inhibitors, integrase inhibitors, fusion inhibitors, and entry inhibitors [55]. A concern with the use of these different medications is how they impact different cellular mechanisms in the context of the immune activation present at therapy initiations and the chronic immune activation that continues in a large percentage of HIV-1-infected persons despite being on HAART $[17,18]$. It may be that certain medications are best used during the induction phase and in patients with chronic immune activation while others have a safety profile making them more suited for the chronic therapy of HIV-1 patients without immune activation [56]. It is possible that exposure to particular medications may correlate with specific genetic lesions that are critical to the development of malignancies in HIV-1-infected patients.

Protease inhibitors (PIs) have been suggested to be agents that prevent malignancy and may even be ripe for repositioning as cancer therapeutics [57]. A number of studies have demonstrated the ability of currently used PIs to inhibit cancer cell growth, and improve sensitivity to chemotherapy and radiation therapy [57]. Protease inhibitors have been demonstrated to promote regression of Kaposi's Sarcoma, lymphomas, fibrosarcomas, multiple myeloma, prostate cancer and several cancer cell lines [58-65]. Despite the encouraging data presented in some in vitro systems and these demonstrations of tumor regression, several studies have reported increased rates of malignancies in patients on PI containing regimens [66,67]. It has also been observed that the aspartyl protease inhibitors used to treat HIV-1 have negative impacts on human immune cells that might be critical for successful immune surveillance such as dendritic cells [68]. Currently used protease inhibitors may also interfere with $\mathrm{T}$ cell function preventing their efficacy in tumor surveillance [69].

Non-nucleotide reverse transcriptase inhibitors (NNRTIs) have also been shown to have anti-proliferative effects on cancer cells [70]. It was therefore unexpected when HIV-1-infected patients on HAART regimens containing NNRTIs containing regimens were observed to have increased rates of cancer [71]. NNRTIs may also have immunesuppressive effects on monocyte derived dendritic cells [72]. It may be that the suppression of dendritic cells and other highly proliferating immune cells is enough to overcome the antiproliferative effect on cancer cells.

Integrase inhibitors have been shown to achieve the highest rates of virological control in HIV-1-infected patients [73]. Integrase inhibitors are suggested to be ideal agents due their limited drug interactions. With this class of drugs being one of the newest options available for patients infected with HIV-1 there is much less information about their potential role in preventing or promoting cancer. One publication that followed a cohort of approximately 2000 patients did describe an increase rate of cancer in patients on integrase inhibitors versus those on HAART regimens not containing integrase inhibitors, but this may reflect a preference by treating physicians to change prior regimens to integrase inhibitor containing regimens to avoid drug interactions when initiating chemotherapy [9]. With there being no clear host homologue to the targeted HIV-1 integrase it may be that this class of medications lacks any negative impacts on host immune tumor surveillance.

\section{Conclusion}

With over 1 million patients in the United States infected with HIV-1 being and new diagnoses each year, we are still unfortunately in the midst of an ongoing epidemic. A current challenge facing the aging HIV-1 positive population is malignancies, in terms of prevalence, morbidity and mortality. Although awareness of the increased prevalence and associated mortality and morbidity of cancer in the HIV-1-infected population is growing, the mechanisms driving this problem are currently unknown. The chronic immune activation present in many HIV-1-infected patients may be involved in increasing the risk of cancer, but the role of decreased immune surveillance and the effects of antiretroviral medication on this surveillance may be critical. Large cohort studies of the relative incidence of cancer in patients on integrase inhibitor therapy compared to those on PI or NNRTI based regimens are currently underway. Further basic science investigation will be critical to understanding the molecular features of these cancers and the impact of various antiretroviral agents on their development.

\section{References}

1. Estimated HIV incidence in the United States 2007-2010 (2012) HIV Surveillance Supplemental Report, CDC 14

2. HIV in the United States (2015) At A Glance, CDC

3. Prejean J, Song R, Hernandez A, Ziebell R, Green T, et al. (2011) Estimated HIV incidence in the United States, 2006-2009. PLoS One 6: e17502.

4. Harrison KM, Song R, Zhang X (2010) Life expectancy after HIV diagnosis based on national HIV surveillance data from 25 states, United States. Journal of acquired immune deficiency syndromes. 53: 124-130.

5. Aberg JA (2012) Aging, inflammation, and HIV infection. Top Antivir Med 20 101-105.

6. Robbins HA, Pfeiffer RM, Shiels MS, Li J, Hall HI, et al. (2015) Excess cancers among HIV-infected people in the United States. J Natl Cancer Inst 107.

7. Simard EP, Engels EA (2010) Cancer as a cause of death among people with AIDS in the United States. Clin Infect Dis 51: 957-962.

8. Griffin DO, Metzger M, Poeth K, Kathy Deng, Arif Dharsee, et al. (2015) Malignancies, Particularly B-cell Lymphomas, are a Frequent Cause of Mortality in HIV-1 Patients Despite HAART. Open Forum Infectious Diseases 2: 147.

9. Griffin DO, Dharsee A, Rico JC, McGowan J (2014) Increasing Deaths Due to Malignancy in HIV+ Patients is Associated with Integrase Inhibitor Therapy. Journal of Health Science 2: 240-247.

10. Rico JC, Schwartz RM, McGowan J, Griffin DO (2016) The Presence of a Malignant Comorbidity is a Significant Predictor of Increased 30-Day Hospital Readmission Rates in HIV-1 Infected Individuals. Journal of AIDS and Clinical Research.

11. Palella FJ, Delaney KM, Moorman AC, Mark O. Loveless, Jack Fuhrer, et al. (1998) Declining morbidity and mortality among patients with advanced human immunodeficiency virus infection. HIV Outpatient Study Investigators. The New England journal of medicine 338: 853-860.

12. Bartlett JA, DeMasi R, Quinn J, Moxham C, Rousseau F (2001) Overview of the effectiveness of triple combination therapy in antiretroviral-naive HIV-1 infected adults. AIDS 15: 1369-1377.

13. Shiels MS, Pfeiffer RM, Gail MH, Hall HI, Li J, et al. (2011) Cancer burden in the HIV-infected population in the United States. J Natl Cancer Inst 103: 753-762.

14. Silverton A, Gunthel C, Adamski M, Mosunjac M, Nguyen ML (2014) Short communication: spectrum of non-Hodgkin lymphoma in an urban Ryan Whitefunded clinic in the established antiretroviral era. AIDS research and human retroviruses 30: 665-669.

15. Hoffmann C, Hentrich M, Gillor D, Behrens G, Jensen B et al. (2014) Hodgkin lymphoma is as common as non-Hodgkin lymphoma in HIV-positive patients with sustained viral suppression and limited immune deficiency: a prospective cohort study. HIV medicine 16: 261-264.

16. Schneider C, Pasqualucci L, Dalla-Favera R (2011) Molecular pathogenesis of diffuse large B-cell lymphoma. Semin Diagn Pathol 28: 167-177.

17. Appay V, Sauce D (2008) Immune activation and inflammation in HIV-1 infection: causes and consequences. J Pathol 214: 231-241.

18. O'Byrne KJ, Dalgleish AG (2001) Chronic immune activation and inflammation as the cause of malignancy. $\mathrm{Br} \mathrm{J}$ Cancer 85: 473-483. 
19. Susnow N, Zeng L, Margineantu D, Hockenbery DM (2009) Bcl-2 family proteins as regulators of oxidative stress. Semin Cancer Biol 19: 42-49.

20. Hanahan D, Weinberg RA (2011) Hallmarks of cancer: the next generation Cell 144: 646-674.

21. Upadhyay R, Hammerich L, Peng P, Brown B, Merad M, et al. (2015) Lymphoma: immune evasion strategies. Cancers (Basel) 7: 736-762.

22. Gajewski TF, Schreiber H, Fu YX (2013) Innate and adaptive immune cells in the tumor microenvironment. Nat Immunol 14: 1014-1022.

23. Lawrence MS, Stojanov P, Mermel CH, Robinson JT, Garraway LA, et al. (2014) Discovery and saturation analysis of cancer genes across 21 tumour types. Nature 505: 495-501.

24. Paiardini M, Frank I, Pandrea I, Apetrei C, Silvestri G (2008) Mucosal immune dysfunction in AIDS pathogenesis. AIDS Rev 10: 36-46.

25. Riemersma SA, Jordanova ES, Schop RF, Philippo K, Looijenga LH, et al. (2000) Extensive genetic alterations of the HLA region, including homozygous deletions of HLA class II genes in B-cell lymphomas arising in immuneprivileged sites. Blood 96: 3569-3577.

26. Thibodeau J, Bourgeois-Daigneault MC, Lapointe R (2012) Targeting the MHC Class II antigen presentation pathway in cancer immunotherapy. Oncoimmunology 1: 908-916.

27. Seliger B, Cabrera T, Garrido F, Ferrone S (2002) HLA class I antigen abnormalities and immune escape by malignant cells. Semin Cancer Biol 12 3-13.

28. Batista FD, Harwood NE (2009) The who, how and where of antigen presentation to B cells. Nat Rev Immunol 9: 15-27.

29. Rimsza LM, Roberts RA, Miller TP, Unger JM, LeBlanc M, et al. (2004) Loss of $\mathrm{MHC}$ class II gene and protein expression in diffuse large B-cell lymphoma is related to decreased tumor immunosurveillance and poor patient survival regardless of other prognostic factors: a follow-up study from the Leukemia and Lymphoma Molecular Profiling Project. Blood 103: 4251-4258.

30. Cycon KA, Rimsza LM, Murphy SP (2009) Alterations in CIITA constitute a common mechanism accounting for downregulation of $\mathrm{MHC}$ class II expression in diffuse large B-cell lymphoma (DLBCL). Experimental hematology 37: 184194.

31. Zeytun A, Hassuneh M, Nagarkatti M, Nagarkatti PS (1997) Fas-Fas ligandbased interactions between tumor cells and tumor-specific cytotoxic $T$ lymphocytes: a lethal two-way street. Blood 90: 1952-1959.

32. Rosenwald A, Wright G, Leroy K, Yu X, Gaulard P, et al. (2003) Molecular diagnosis of primary mediastinal $B$ cell lymphoma identifies a clinically favorable subgroup of diffuse large B cell lymphoma related to Hodgkin lymphoma. The Journal of experimental medicine 198: 851-862.

33. Marshall NA, Christie LE, Munro LR, Culligan DJ, Johnston PW, et al. (2004) Immunosuppressive regulatory $T$ cells are abundant in the reactive lymphocytes of Hodgkin lymphoma. Blood 103: 1755-1762.

34. Yang ZZ, Novak AJ, Stenson MJ, Witzig TE, Ansell SM (2006) Intratumora CD4+ CD25+ regulatory T-cell-mediated suppression of infiltrating CD4+ T cells in B-cell non-Hodgkin lymphoma. Blood 107: 3639-3646.

35. Mittal S, Marshall NA, Duncan L, Culligan DJ, Barker RN, et al. (2008) Local and systemic induction of CD4+ CD25+ regulatory T-cell population by nonHodgkin lymphoma. Blood 111: 5359-5370.

36. Steidl C, Lee T, Shah SP, Farinha P, Han G, et al. (2010) Tumor-associated macrophages and survival in classic Hodgkin's lymphoma. N Engl J Med 362: 875-885.

37. Wada N, Zaki MA, Hori Y, Hashimoto K, Tsukaguchi M, et al. (2012) Tumourassociated macrophages in diffuse large B-cell lymphoma: a study of the Osaka Lymphoma Study Group. Histopathology 60: 313-319.

38. Wu X, Schulte BC, Zhou Y, Haribhai D, Mackinnon AC, et al. (2014) Depletion of M2-like tumor-associated macrophages delays cutaneous T-cell lymphoma development in vivo. J Invest Dermatol 134: 2814-2822.

39. Gandhi MK, Moll G, Smith C, Dua U, Lambley E, et al. (2007) Galectin-1 mediated suppression of Epstein-Barr virus specific T-cell immunity in classic Hodgkin lymphoma. Blood 110: 1326-1329.

40. von Bergwelt-Baildon MS, Popov A, Saric T, Chemnitz J, Classen S, et al. (2006) CD25 and indoleamine 2,3-dioxygenase are up-regulated by prostaglandin E2 and expressed by tumor-associated dendritic cells in vivo: additional mechanisms of T-cell inhibition. Blood 108: 228-237.

41. Chemnitz JM, Driesen J, Classen S, Riley JL, Debey S, et al. (2006) Prostaglandin E2 impairs CD4+ T cell activation by inhibition of lck: implications in Hodgkin's lymphoma. Cancer Res 66: 1114-1122.

42. Hedlund M, StenqvistAC, Nagaeva O, Kjellberg L, Wulff MO, et al. (2009) Human placenta expresses and secretes NKG2D ligands via exosomes that downmodulate the cognate receptor expression: evidence for immunosuppressive function. J Immunol. 183: 340-351.

43. Hedlund M, Nagaeva O, Kargl D, Baranov V, Mincheva-Nilsson L (2011) Thermal- and oxidative stress causes enhanced release of NKG2D ligandbearing immunosuppressive exosomes in leukemia/lymphoma $\mathrm{T}$ and $\mathrm{B}$ cells. PLoS One 6: e16899.

44. Pizzolo G, Ambrosetti A, Vinante F, Chilosi M, Semenzato G (1991) Serum interleukin-2 receptor as index of tumor burden in hairy cell leukemia. Blood 77: $2540-2542$.

45. Ambrosetti A, Nadali G, Vinante F, Stefano Carlini, Dino Veneri, et al. (1993) Serum levels of soluble interleukin-2 receptor in Hodgkin disease. Relationship with clinical stage, tumor burden, and treatment outcome. Cancer 72: 201-206.

46. Voorzanger N, Touitou R, Garcia E, Delecluse HJ, Rousset F, et al. (1996) Interleukin (IL)-10 and IL-6 are produced in vivo by non-Hodgkin's lymphoma cells and act as cooperative growth factors. Cancer Res 56: 5499-5505.

47. Cui X, Zhang L, Magli AR, Catera R, Yan XJ, et al. (2016) Cytoplasmic myosinexposed apoptotic cells appear with caspase- 3 activation and enhance CLL cel viability. Leukemia 30: 74-85

48. Griffin DO, Rothstein TL (2012) Human "orchestrator" CD11b(+) B1 cells spontaneously secrete interleukin-10 and regulate T-cell activity. Molecular medicine 18: 1003-1008.

49. Iwata Y, Matsushita T, Horikawa M, Dilillo DJ, Yanaba K, et al. (2011) Characterization of a rare IL-10-competent B-cell subset in humans that parallels mouse regulatory B10 cells. Blood 117: 530-541.

50. DiLillo DJ, Weinberg JB, Yoshizaki A, Horikawa M, Bryant JM, et al. (2013) Chronic lymphocytic leukemia and regulatory B cells share IL-10 competence and immunosuppressive function. Leukemia 27: 170-182.

51. Griffin DO, Rothstein TL (2012) Human "orchestrator" CD11b(+) B1 cells spontaneously secrete interleukin-10 and regulate T-cell activity. Mol Med 18 1003-1008.

52. Cui X, Zhang L, Magli AR, Catera R, Yan XJ, et al. (2016) Cytoplasmic myosinexposed apoptotic cells appear with caspase-3 activation and enhance CLL cell viability. Leukemia 30: 74-85

53. Yanaba K, Bouaziz JD, Haas KM, Poe JC, Fujimoto M, et al. (2008) A regulatory $B$ cell subset with a unique CD1dhiCD5+ phenotype controls $T$ cell-dependent inflammatory responses. Immunity 28: 639-650.

54. Yang ZZ, Grote DM, Ziesmer SC, Xiu B, Yates NR, et al. (2013) Soluble and membrane-bound TGF- $\left.\right|^{2}$-mediated regulation of intratumoral $T$ cell differentiation and function in B-cell non-Hodgkin lymphoma. PLoS One 8 : e59456.

55. McColl DJ, Chen X (2010) Strand transfer inhibitors of HIV-1 integrase: bringing IN a new era of antiretroviral therapy. Antiviral Res 85: 101-118.

56. Wutzler P, Thust R (2001) Genetic risks of antiviral nucleoside analogues--a survey. Antiviral Res 49: 55-74.

57. Bernstein WB, Dennis PA (2008) Repositioning HIV protease inhibitors as cancer therapeutics. Curr Opin HIV AIDS 3: 666-675.

58. Martinelli C, Zazzi M, Ambu S, Bartolozzi D, Corsi P, et al. (1998) Complete regression of AIDS-related Kaposi's sarcoma-associated human herpesvirus-8 during therapy with indinavir. AIDS 12: 1717-1719.

59. Niehues T, Horneff G, Megahed M, Schroten H, Wahn V (1999) Complete regression of AIDS-related Kaposi's sarcoma in a child treated with highly active antiretroviral therapy. AIDS 13: 1148-1149.

60. Monini P, Sgadari C, Toschi E, Barillari G, Ensoli B (2004) Antitumour effects of antiretroviral therapy. Nat Rev Cancer 4: 861-875.

61. Pati S, Pelser CB, Dufraine J, Bryant JL, Reitz MS Jr, et al. (2002) Antitumorigenic effects of HIV protease inhibitor ritonavir: inhibition of Kaposi sarcoma. Blood 99: 3771-3779. 
Citation: Griffin DO (2016) The Challenge of Malignancies in HIV-1, Beyond Immune Activation and Back to Decreased Immune Surveillance. J AIDS Clin Res 7: 535. doi:10.4172/2155-6113.1000535

62. Gaedicke S, Firat-Geier E, Constantiniu O, Lucchiari-Hartz M, Freudenberg M et al. (2002) Antitumor effect of the human immunodeficiency virus protease inhibitor ritonavir: induction of tumor-cell apoptosis associated with perturbation of proteasomal proteolysis. Cancer Res 62: 6901-6908.

63. Yang Y, Ikezoe T, Nishioka C, Bandobashi K, Takeuchi T, et al. (2006) NFV, an HIV-1 protease inhibitor, induces growth arrest, reduced Akt signalling, apoptosis and docetaxel sensitisation in NSCLC cell lines. British journal of cancer 95: 1653-1662.

64. Yang Y, Ikezoe T, Takeuchi T, Adachi Y, Ohtsuki Y, et al. (2005) HIV-1 protease inhibitor induces growth arrest and apoptosis of human prostate cancer LNCaP cells in vitro and in vivo in conjunction with blockade of androgen receptor STAT3 and AKT signaling. Cancer Sci 96: 425-433.

65. Ikezoe T, Saito T, Bandobashi K, Yang Y, Koeffler HP, et al. (2004) HIV1 protease inhibitor induces growth arrest and apoptosis of human multiple myeloma cells via inactivation of signal transducer and activator of transcription 3 and extracellular signal-regulated kinase 1/2. Mol Cancer Ther. 3: 473-479.

66. Bruyand M, Ryom L, Shepherd L, Fatkenheuer G, Grulich A, et al. (2015) Cancer risk and use of protease inhibitor or non-nucleoside reverse transcriptase inhibitor-based combination antiretroviral therapy: the D: A: D study. Journal of acquired immune deficiency syndromes $68:$ 568-577.

67. Chao C, Leyden WA, Xu L, Horberg MA, Klein D, et al. (2012) Exposure to antiretroviral therapy and risk of cancer in HIV-infected persons. AIDS 26 : 2223-2231.
68. Gruber A, Wheat JC, Kuhen KL, Looney DJ, Wong-Staal F (2001) Differential effects of HIV-1 protease inhibitors on dendritic cell immunophenotype and function. J Biol Chem 276: 47840-47843.

69. Sarin A, Adams DH, Henkart PA (1993) Protease inhibitors selectively block $T$ cell receptor-triggered programmed cell death in a murine $T$ cell hybridoma and activated peripheral $T$ cells. The Journal of experimental medicine 178: 1693-1700.

70. Hecht M, Erber S, Harrer T, Klinker H, Roth T, et al. (2015) Efavirenz Has the Highest Anti-Proliferative Effect of Non-Nucleoside Reverse Transcriptase Inhibitors against Pancreatic Cancer Cells. PloS one 10: e0130277.

71. Powles T, Robinson D, Stebbing J, Jonathan Shamash, Mark Nelson, et al (2009) Highly active antiretroviral therapy and the incidence of non-AIDSdefining cancers in people with HIV infection. Journal of clinical oncology: official journal of the American Society of Clinical Oncology 27: 884-890.

72. Van Herrewege Y, Penne L, Vereecken C, Katrien Fransen, Guido van der Groen, et al. (2002) Activity of reverse transcriptase inhibitors in monocytederived dendritic cells: a possible in vitro model for post-exposure prophylaxis of sexual HIV transmission. AIDS research and human retroviruses 18: 10911102.

73. Torres HA, Rallapalli V, Saxena A, Granwehr BP, Viola GM, et al. (2014) Efficacy and safety of antiretrovirals in HIV-infected patients with cancer. Clin Microbiol Infect 20: 0672-679. 